\title{
KIF20A expression as a prognostic indicator and its possible involvement in the proliferation of ovarian clear-cell carcinoma cells
}

\author{
YOSUKE KAWAI $^{1,2}$, KIYOSUMI SHIBATA ${ }^{3}$, JUN SAKATA ${ }^{1}$, SHIRO SUZUKI $^{1}$, FUMI UTSUMI $^{1}$, \\ KAORU NIIMI ${ }^{1}$, RYUICHIRO SEKIYA ${ }^{3}$, TAKESHI SENGA ${ }^{4}$, \\ FUMITAKA KIKKAWA $^{1}$ and HIROAKI KAJIYAMA ${ }^{1}$
}

\author{
${ }^{1}$ Department of Obstetrics and Gynecology, Graduate School of Medicine, Nagoya University, Nagoya 466-8550; \\ ${ }^{2}$ Department of Obstetrics and Gynecology, Toyohashi Municipal Hospital, Toyohashi 441-8570; ${ }^{3}$ Department of \\ Obstetrics and Gynecology, Banbuntane Hotokukai Hospital, Fujita Health University, Nagoya 454-8509; \\ ${ }^{4}$ Department of Internal Medicine, Yahagigawa Hospital, Aichi 444-1164, Japan
}

Received September 24, 2017; Accepted March 16, 2018

DOI: $10.3892 / o r .2018 .6401$

\begin{abstract}
Kinesin family member 20A (KIF20A), which is involved in cytokinesis and intracellular transportation, has been recently reported to be upregulated in several malignancies and may contribute to chemotherapeutic resistance. We examined the distribution and expression of KIF20A in clear-cell carcinoma (CCC) of the ovary to elucidate its clinical significance and molecular mechanism. Paraffin sections from ovarian CCC tissues ( $\mathrm{N}=43$ ) were immunostained with KIF20A antibody, and the staining intensities were semi-quantitatively evaluated. Furthermore, we investigated whether silencing of KIF20A contributes to the proliferation-inhibitory potential using CCC cells. During the observational period, 18 patients (41.9\%) developed recurrence. The median time to recurrence was 11.5 months. Patients in the high KIF20A expression group showed poorer progression-free survival (PFS) and overall survival (OS) than those in the low expression group $(\mathrm{P}=0.0443$ and $\mathrm{P}=0.0478$, respectively). In multivariable analyses, KIF20A expression was also a significantly independent indicator of PFS and a marginally significant indicator of OS [PFS: HR (high vs. low), 5.488; 95\% CI, 1.410-24.772 ( $\mathrm{P}=0.0136$ ); OS: $\mathrm{HR}, 2.835$; 95\% CI, 0.854-11.035, $(\mathrm{P}=0.0897)]$. In in vitro studies, the ovarian CCC cell proliferation was significantly decreased by KIF20A silencing or in the presence of KIF20A
\end{abstract}

Correspondence to: Dr Hiroaki Kajiyama, Department of Obstetrics and Gynecology, Graduate School of Medicine, Nagoya University, Truma-cho 65, Showa-ku, Nagoya 466-8550, Japan E-mail: kajiyama@med.nagoya-u.ac.jp

Abbreviations: EOC, epithelial ovarian cancer; CCC, clear-cell carcinoma; FIGO, International Federation of Gynecology and Obstetrics; OS, overall survival; PFS, progression-free survival

Key words: epithelial ovarian carcinoma, clear-cell carcinoma, KIF20A, immunohistochemical staining, prognostic indicator, cell proliferation, cell cycle inhibitor in CCC cells. Cell cycle G2/M arrest and a higher apoptosis-induced fraction were more frequently observed in si-KIF20A-transfected CCC cells than in the control cells. Although the present study was preliminary, these data indicate the possible involvement of KIF20A in the proliferation of CCC, suggesting that targeting this molecule may contribute to reversing the malignant potential consequently affecting the oncologic outcome of CCC patients.

\section{Introduction}

Epithelial ovarian cancer (EOC) is a major cause of cancer-related mortality among gynecologic malignancies. Based on the fact that EOC frequently remains clinically silent, most patients have microscopically or macroscopically metastatic peritoneal lesions at diagnosis (1). However, EOC is in general a heterogeneous tumor that contains various histologic types, including serous, clear-cell, endometrioid, mucinous and other types of adenocarcinoma. The clinical outcomes and biological hallmarks are different among these histological subtypes. This diversity makes it complicated to understand and analyze EOC (2). Ovarian clear-cell carcinoma (CCC) is a comparatively rare tumor in Western countries, representing less than $10 \%$ of all epithelial ovarian cancer (EOC) cases diagnosed (3). However, the incidence of this tumor was estimated to make it a common pathological type among EOC cases in East Asia (4). In particular, CCC is the second most frequent subtype of EOC, accounting for $24.8 \%$ of all malignant ovarian neoplasms diagnosed in Japan (5). Clinically, this tumor is known to be diagnosed at an early stage, and shows frequent unilateral occurrence, association with endometriosis, and comorbidity with thromboembolism (3,6-8). In addition, based on recent larger-scale studies, a poorer oncologic outcome is exhibited by patients with advanced-stage CCC compared with those with a serous histology, reflecting its potential chemoresistance to conventional platinum-based compounds $(9,10)$. Therefore, it is crucial to determine prognostic biomarkers of CCC to 
develop strategies for improving the clinical outcome and/or monitoring the tumor status.

Kinesin family member 20A (KIF20A), a member of the kinesin superfamily- 6 , is a microtubule-associated motor protein that is required for cell cycle mitosis $(11,12)$. This molecule was initially revealed to localize to the Golgi apparatus and participate in organelle dynamics by interacting with the GTP-bound form of Rab6 (13). In non-cancerous normal tissues, KIF20A is reported to be expressed in fetal liver, adult bone marrow and thymus, whereas low levels are found in the placenta and heart $(14,15)$. On the other hand, according to previous studies, it was shown to be overexpressed in various malignancies, including lung and breast cancer $(15,18)$. With regard to malignant cellular functions, KIF20A has been reported to be involved in proliferation, migration, invasiveness and angiogenesis (15,19-21). In addition, a recent study also demonstrated the possible involvement of KIF20A in the resistance of breast cancer to paclitaxel (22). From the clinical point of view, KIF20A expression was found to be associated with poor oncologic outcome in a number of human malignancies, including breast, nasopharyngeal, hepatocellular and uterine cervical carcinoma (23-26).

To the best of our knowledge, no published study has examined KIF20A expression in ovarian CCC. These clinical and molecular backgrounds led us to hypothesize that KIF20A plays a central role in the progression of $\mathrm{CCC}$, and that positive KIF20A expression may be a valuable indicator to predict an unfavorable oncologic outcome in patients with CCC. In the present study, we explored the relationship between KIF20A expression and the prognosis of ovarian CCC patients, and we analyzed the functions of KIF20A in CCC cell growth.

\section{Materials and methods}

Cell culture. One human pancreatic cancer cell line (PANC1: positive control) (15) and seven EOC cell lines (ES-2, TOV-21G, RMG-I, RMG-II, SKOV3, OV-90 and KOC-7C) were maintained in RPMI-1640 medium with $10 \%$ fetal bovine serum (FBS) and penicillin/streptomycin. These cell lines except for RMG-I and RMG-II cells were purchased from the American Type Culture Collection (ATCC; Manassas, VA, USA) in 2012-2013. RMG-I and RMG-II cells were generously donated by Fujita Health University (Toyoake, Japan). These cell lines were maintained in RPMI-1640 medium (Sigma-Aldrich; Merck KGaA, Darmstadt, Germany) supplemented with $10 \%$ FBS and penicillin-streptomycin at $37^{\circ} \mathrm{C}$ in a humidified atmosphere of $5 \% \mathrm{CO}_{2}$.

Inhibition of KIF20A by small interfering RNA (siRNA). To produce KIF20A-knockdown cells, ES-2 and SKOV3 cells were transfected with either a pool of small interfering RNA (siRNA) oligonucleotides specific to human KIF20A (final concentration, 50 nmol/1; sc-91657; Santa Cruz Biotechnology, Santa Cruz, CA, USA) or control siRNA (5'-CUUACGCUG AGUACUUCGATT-3': Sigma-Aldrich; Merck KGaA) using GenePORTER 2 Transfection reagent (Gene Therapy Systems Inc., San Diego, CA, USA). KIF20A siRNA (sc-91657) is a pool of 3 different siRNA duplexes: sc-91657A (sense, 5'-CUGUGA AGGAGAUGGUAAATT-3'), sc-91657B (sense, 5'-GCAAUC CCUAUGUGAAAGATT-3') and sc-91657C (sense, 5'-GUU
CCUGCAUGAUUGUCAATT-3'). After overnight incubation at $37^{\circ} \mathrm{C}$, the culture medium was replaced with fresh complete medium containing 10\% FBS. Cells were harvested after $48 \mathrm{~h}$ and solubilized for western blot analysis of KIF20A silencing.

Western blot analysis. To examined the expression of KIF20A in seven EOC cell lines (CCC: ES-2, TOV-21G, RMG-I, RMG-II, KOC-7C cells: non-CCC SKOV3, OV-90 cells) or knockdown efficacy of the siRNA experiment (ES-2 and SKOV3 cells), western blotting was performed. The experimental procedure of western blotting was previously described (27). As primary antibodies, we used anti-KIF20A (diluted 1:100; cat. no. sc-374508; Santa Cruz Biotechnology) and anti- $\beta$-actin antibodies (diluted 1:3,000; cat. no. 017-24573; Wako Pure Chemical Industries Ltd., Osaka, Japan). The primary antibodies were washed in $0.05 \%$ Tween-20/PBS, and then incubated with horseradish peroxidase-conjugated secondary antibody (diluted 1:3,000; cat. no. A90-116P; Bethyl Laboratories Inc., Montgomery, TX, USA). Proteins were visualized using enhanced chemiluminescence reagent (Amersham Pharmacia Biotech, Piscataway, NJ, USA). The ratio of the intensity of KIF20A-positive bands to the intensity of $\beta$-actin-positive ones was used to compare the relative expression levels of these proteins in the KIF20A- and control-siRNA transfected lines. The intensities of the bands were semi-quantified by ImageJ software (version 1.51k; National Institutes of Health, Bethesda, MD, USA) (https://imagej.nih.gov/ij/).

Cell proliferation assay. To determine the effect of KIF20A on cell proliferation, ES2 and SKOV3 cells transfected with KIF20A-siRNA or control siRNA were seeded onto 96-well plates at a density of $1 \times 10^{3}$ cells/well. Then, at time-points of $0,24,48$ and $72 \mathrm{~h}$, the cell viability rate was assessed using Cell Counting Kit-8 (CCK-8; Dojindo Molecular Technologies, Inc., Rockville, MD, USA). Ten microliters of CCK-8 solution was added to each well and the cells were incubated for another $2 \mathrm{~h}$ and then a microplate reader (ELx808; BioTek Instruments Japan, Tokyo, Japan) was used to measure the absorbance of each well at $450 \mathrm{~nm}$. All experiments were independently repeated three times. Paprotrain (Tocris Bioscience, Bristol, UK) is a cell-permeable acrylonitrile compound that inhibits the kinesin- 6 family member KIF20A. We investigated the effect of Paprotrain on cell proliferation using pretreatment at doses of 0,25 and $100 \mu \mathrm{M}$ in the ES2 and SKOV3 cells.

Apoptosis assay. The percentage of apoptotic cells was assayed using the Annexin V-FLUOS Staining kit (Roche Life Science, Indianapolis, IN, USA). Briefly, $1 \times 10^{5}$ ES- 2 cells were seeded in 6-well plates and cultured for $24 \mathrm{~h}$. The cells were collected and resuspended in $100 \mu \mathrm{l}$ of binding buffer. Then, the cells were incubated with $5 \mu \mathrm{l}$ of FITC-Annexin V in the dark for $15 \mathrm{~min}$ at room temperature. Subsequently, $5 \mu \mathrm{l}$ of PI was added and incubated with the cells for $20 \mathrm{~min}$ at room temperature in the dark. Finally, the cell samples were examined in a flow cytometer. Each assessment of proliferation and apoptosis was repeated three times.

Cell cycle distribution. For cell cycle analysis, ES2 and SKOV3 cells were treated with or without S109 for $24 \mathrm{~h}$. Then, the cells were collected, fixed in $70 \%$ ethanol, washed twice 
with phosphate-buffered saline (PBS), and then stained with propidium iodide (PI) solution containing $25 \mu \mathrm{g} / \mathrm{ml}$ of RNAse and $50 \mu \mathrm{g} / \mathrm{ml}$ of PI in the dark for $30 \mathrm{~min}$. Subsequently, the cells were assayed with FACSCalibur (Becton-Dickinson, Franklin Lakes, NJ, USA) and analyzed using CellQuest Pro software (Becton-Dickinson).

Patients and immunohistochemical staining. Forty-three human ovarian CCC tissues were obtained from patients who underwent surgical treatment at the Nagoya University Hospital between 1993 and 2006 after providing informed consent. To ascertain the histological types, we adopted the World Health Organization (WHO) classification criteria. The clinical stage was assigned according to the International Federation of Gynecology and Obstetrics (FIGO) staging system $(28,29)$. This study was approved by the Ethics Committee of Nagoya University.

Formalin-fixed, paraffin-embedded tissue sections were cut at a thickness of $4 \mu \mathrm{m}$. For heat-induced epitope retrieval, deparaffinized sections in $0.01 \mathrm{M}$ citrate buffer (Target Retrieval Solution, pH 6.1; Dako, Tokyo Japan) were heated three times at $90^{\circ} \mathrm{C}$ for 5 min using a microwave oven. Sections were incubated at $4^{\circ} \mathrm{C}$ for $12 \mathrm{~h}$ with primary antibody (anti-rabbit KIF20A polyclonal, at a 1:100 dilution; Santa Cruz Biotechnology). The sections were rinsed and incubated for 30 min with biotinylated anti-rabbit IgG antibody (cat. no. 424022; Nichirei Biosciences Inc., Tokyo, Japan). The immunoreactive staining was processed using the peroxidase-anti-peroxidase method according to the manufacturer's instructions (Dako GmbH, Hamburg, Germany). To detect the reaction, 3,3'-diaminobenzidine tetrachloride (DAB) chromogen solution was used. After rinsing in water for $30 \mathrm{~min}$, the sections were counterstained with hematoxylin and then dehydrated. Finally, they were mounted in mounting medium for examination. The specificity of the antibody was determined using a non-specific immunoglobulin IgG (diluted 1:100; cat. no. sc-2025; Santa Cruz Biotechnology) at the same concentration.

Evaluation of immunohistochemical staining. For the evaluation of the results of immunohistochemical staining, 10 fields of each specimen were selected and evaluated with both low- (magnification, x100) and high-power microscopy (magnification, $\mathrm{x} 400$ ). Two investigators assessed the slides without knowledge of the clinicopathological features and were blinded to each other's evaluation. The two investigators were in agreement on all the slides examined. Based on the immunostaining activity, a semi-quantitative score was assigned according to the intensity and area of the stained cells, as previously described (30). For the evaluation of KIF20A expression, the staining intensity was scored as 0 (negative-weak), 1 (medium), 2 (strong), or 3 (very strong). The percentage of the staining area was scored as $0(0-10 \%)$, $1(11-50 \%)$, and $2(51-100 \%)$ relative to the total tumor area. The sum of the staining intensity and area scores was calculated as the final score (0-5) for KIF20A. Tumors with a final score of 0-1 and 2-5, were classified as showing low and high expression, respectively.

Survival analyses. The distributions of clinicopathological factors were statistically assessed using the Chi-square test or the Fisher's exact test. The progression-free survival (PFS) was defined as the time interval between the date of surgery and the date of the last follow-up or recurrence/progression. The overall survival (OS) was defined as the time interval between the date of surgery and the date of the last follow-up or death from any cause. The survival curves were compared employing the log-rank test. Survival analysis was conducted using the Kaplan-Meier method. The prognostic significance of KIF20A expression concerning other clinicopathological variables was assessed using the univariable and multivariable Cox's proportional hazard's analyses. All statistical analyses were performed with the JMP Pro Ver. 10.0 (SAS Institute Japan Ltd., Tokyo, Japan). A P $<0.05$ was considered to indicate a statistically significant result.

Statistical analysis. All data are expressed as the mean \pm SD. Data were calculated from at least three independent experiments. The significance of differences was analyzed by Student's t-test or one-way ANOVA. A value of $\mathrm{P}<0.05$ was considered to indicate a statistically significant result.

\section{Results}

Patient characteristics. We first explored KIF20A expression and its possible involvement in the oncologic outcome of patients with ovarian CCC using immunohistochemical analysis. Patient characteristics are detailed in Table I. The median (range) age was 52 (27-71) years. The distributions of the FIGO stage were $58.1 \%$ (25/43) stage I, $18.6 \%(8 / 43)$ stage II, $18.6 \%(8 / 43)$ stage III, and $4.7 \%$ (2/43) stage IV. Of all patients, $31(72.1 \%)$ underwent complete surgery with surgical staging, and $37(86.0 \%)$ had no residual tumor at the initial surgery. Thirty-seven patients $(86.0 \%)$ were administered postoperative chemotherapy. Six patients did not undergo postoperative chemotherapy owing to their strong wishes or severe complications. Of the 43 CCC patients, the distributions of KIF20A staining expression was as follows: 7 (score, 0), 11 (score, 1), 7 (score, 2), 6 (score, 3), 6 (score, 4) and 6 (score, 5). KIF20A immunoexpression was classified into the two scoring groups as described above [low (score 0-1) and high (score 2-5)]. Representative images of tissue samples for each immunohistochemical staining score are shown in Fig. 1. For the distribution of age and chemotherapy frequency, there was a significant difference between the two groups (Table I).

Oncologic outcome according to KIF20A expression. The median follow-up duration was 64.9 , ranging from 4.3 to 159.3 months in all patients. During this period, 18 patients (41.9\%) developed recurrence. The median time to recurrence was 11.5 months. The 5-year PFS and OS rates of all CCC patients were 60.0 and $63.5 \%$, respectively. Patients in the high KIF20A expression group showed poorer PFS and OS than those in the low expression group [PFS (log-rank: $\mathrm{P}=0.0443$; Cox hazard: $\mathrm{P}=0.0374$ ) and $\mathrm{OS}$ (log-rank: $\mathrm{P}=0.0478$, Cox hazard: $\mathrm{P}=0.0411$ ), respectively] (Fig. 2). Confining analysis to stage I patients with $\mathrm{CCC}$, similar prognostic tendencies were observed (PFS: $\mathrm{P}=0.0484$; OS: $\mathrm{P}=0.0486$ ) (Fig. 3).

Univariable and multivariable analyses. We subsequently performed univariable and multivariable Cox 

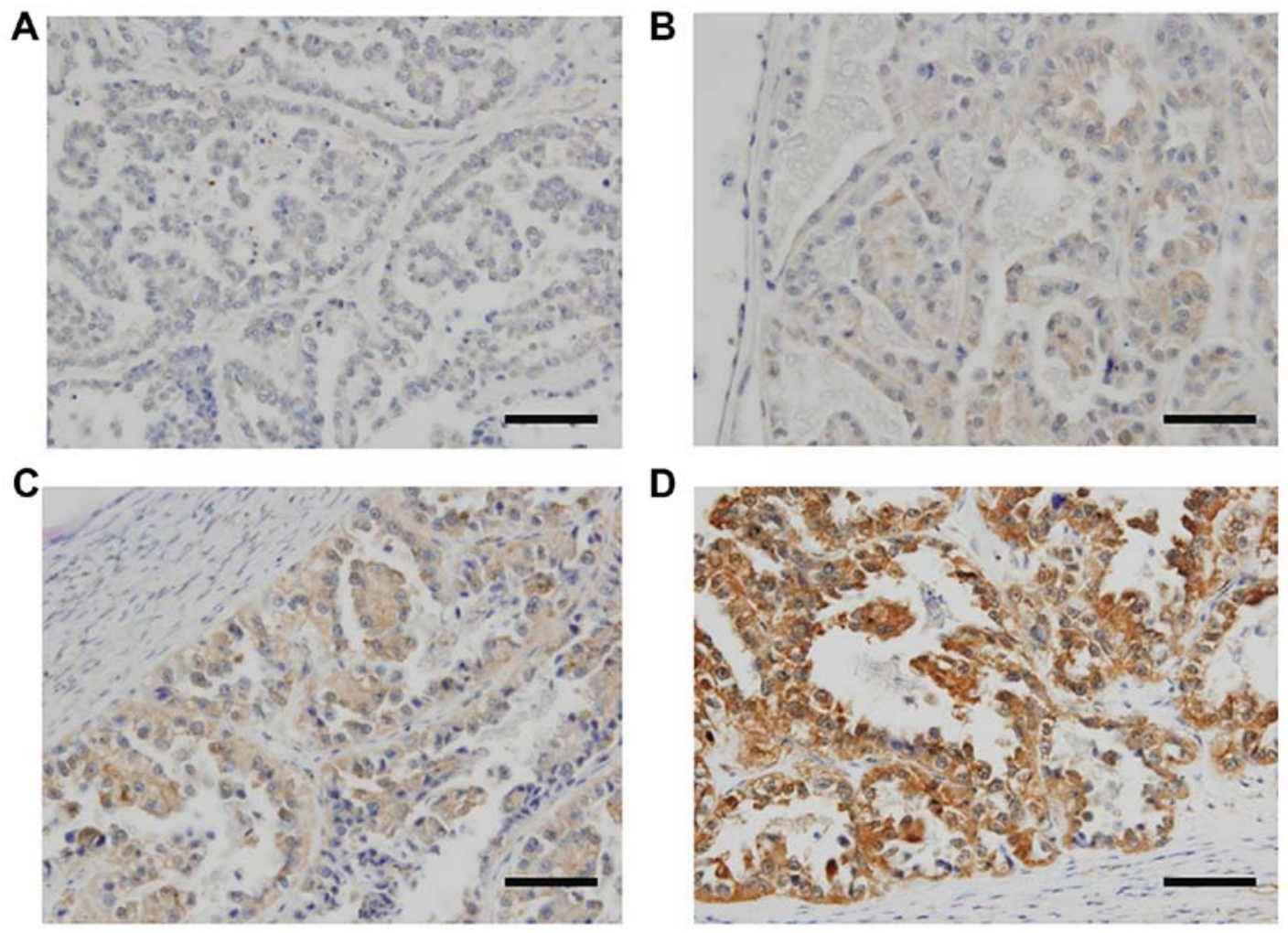

Figure 1. Immunoreactive KIF20A expression in ovarian CCC tissues (paraffin sections). Based on the KIF20A immunostaining activity, a semi-quantitative score was assigned according to the intensity and area of the stained cells, as described in the text. (A) Negative-weak (score 0, low); (B) medium (score 1, low); (C) medium (score 3, high); (D) very strong (score 5, high); (magnification, x100). Scale bars, $100 \mu \mathrm{m}$.
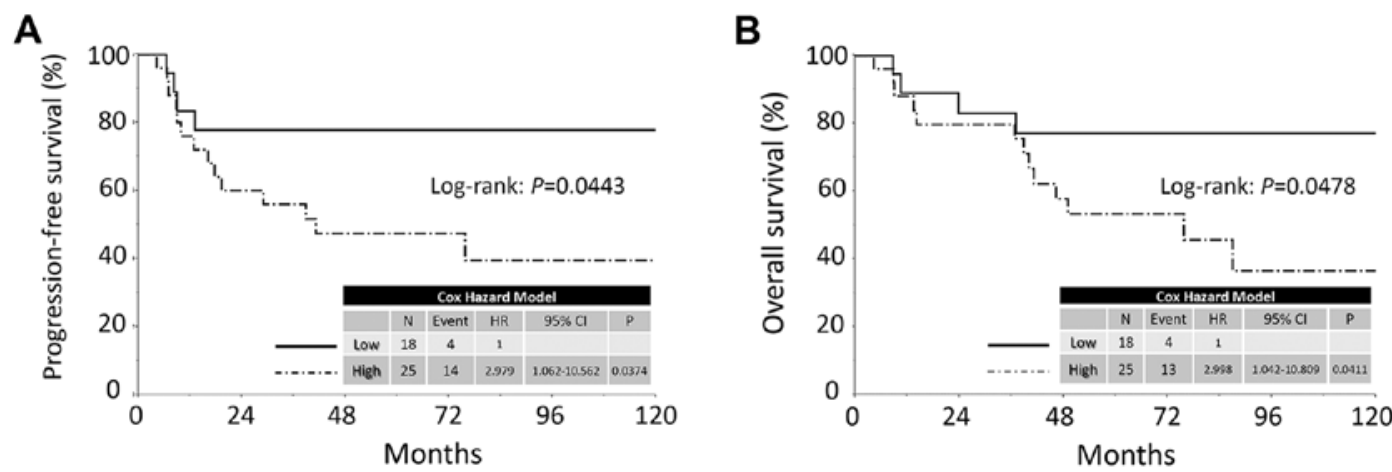

Figure 2. Kaplan-Meier progression-free survival curves for ovarian CCC patients according to the immunoreactivity of KIF20A. (A) PFS. Solid line indicates low KIF20A expression ( $\mathrm{N}=18$ ). Dashed line represents high KIF20A immunoexpression (N=25). Patients with positive KIF20A expression showed a significantly poorer PFS ( $\mathrm{P}=0.0443)$. (B) OS. Solid line indicates low KIF20A expression ( $\mathrm{N}=18)$. Dashed line represents high KIF20A immunoexpression ( $\mathrm{N}=25)$. Patients positive for KIF20A expression showed a significantly poorer OS $(\mathrm{P}=0.0478)$. PFS, progression-free survival; OS, overall survival.

proportional analyses regarding PFS/OS, including age $(\leq 50$ vs. $>50$ years), FIGO stage (I+II vs. III+IV), preoperative CA-125 values ( $\leq 35$ vs. $>35 \mathrm{U} / \mathrm{ml}$ ), volume of ascites $(\leq 100$ vs. $>100 \mathrm{ml}$ ), type of surgery (standard surgery with full staging vs. other surgery), residual tumor (yes vs. no), chemotherapy (platinum-based vs. taxane plus platinum) and KIF20A immunoreactivity (low vs. high) (Table II). In the univariable analyses, the preoperative CA-125 value, volume of ascites, FIGO stage, residual tumor presence and KIF20A expression were significant prognostic indicators of a poor PFS. In addition, KIF20A expression was found to be a marginally significant prognostic indicator of a poor $\mathrm{OS}(\mathrm{P}=0.0512)$. In general, age was considered to be an important clinical factor influencing patient survival. On the other hand, the preoperative CA-125 value, volume of ascites, and residual tumor were strongly correlated with the stage. To avoid multicollinearity, we entered the age, stage and KIF20A expression factors into the multivariable analyses. In multivariable analyses, the KIF20A expression was also a significantly independent indicator for PFS and a marginal-significant indicator for OS [PFS: HR (high vs. low), 5.488 ; 95\% CI, 1.410-24.772 ( $\mathrm{P}=0.0136$ ); OS: HR, 2.835; 95\% CI, 0.854-11.035 ( $\mathrm{P}=0.0897)]$ (Table II).

KIF20A expression in the deceased ovarian CCC patients. Table III shows the clinical backgrounds of the 17 deceased patients. The high-level expression of KIF20A was observed 
Table I. Relationship between the expression of KIF20A and clinicopathologic parameters.

\begin{tabular}{|c|c|c|c|c|c|c|}
\hline & \multicolumn{5}{|c|}{ KIF20A expression } & \multirow[b]{3}{*}{ P-value } \\
\hline & \multirow{2}{*}{$\frac{\text { Total }}{\mathrm{N}}$} & \multicolumn{2}{|c|}{ Low } & \multicolumn{2}{|c|}{ High } & \\
\hline & & $\mathrm{N}$ & $\%$ & $\mathrm{~N}$ & $\%$ & \\
\hline Total no. of cases & 43 & 18 & & 25 & & \\
\hline \multicolumn{7}{|l|}{ Age (years) } \\
\hline$\leq 50$ & 21 & 14 & 77.8 & 7 & 28.0 & \multirow[t]{2}{*}{0.001} \\
\hline$>50$ & 22 & 4 & 22.2 & 18 & 72.0 & \\
\hline \multicolumn{7}{|l|}{ FIGO stage } \\
\hline I & 25 & 12 & 66.7 & 13 & 52.0 & \multirow[t]{4}{*}{0.697} \\
\hline II & 8 & 3 & 16.7 & 5 & 20.0 & \\
\hline III & 8 & 2 & 11.1 & 6 & 24.0 & \\
\hline IV & 2 & 1 & 5.6 & 1 & 4.0 & \\
\hline \multicolumn{7}{|l|}{ Preoperative CA-125 value (U/ml) } \\
\hline$\leq 35$ & 32 & 12 & 66.7 & 20 & 80.0 & \multirow[t]{2}{*}{0.323} \\
\hline$>35$ & 11 & 6 & 33.3 & 5 & 20.0 & \\
\hline \multicolumn{7}{|l|}{ Ascites volume (ml) } \\
\hline$<100$ & 23 & 11 & 61.1 & 12 & 48.0 & \multirow[t]{4}{*}{0.245} \\
\hline $100-1,000$ & 5 & 0 & 0.0 & 5 & 20.0 & \\
\hline$>1,000$ & 7 & 3 & 16.7 & 4 & 16.0 & \\
\hline NA & 8 & 4 & 22.2 & 4 & 16.0 & \\
\hline \multicolumn{7}{|l|}{ Surgery } \\
\hline Standard surgery + full staging & 31 & 14 & 77.8 & 17 & 68.0 & \multirow[t]{5}{*}{0.417} \\
\hline Standard surgery ${ }^{\mathrm{a}}$ & 6 & 2 & 11.1 & 4 & 16.0 & \\
\hline $\mathrm{USO} / \mathrm{BSO} \pm \mathrm{OM}$ & 2 & 1 & 5.6 & 1 & 4.0 & \\
\hline Debulking surgery & 1 & 1 & 5.6 & 0 & 0.0 & \\
\hline Exploratory & 3 & 0 & 0.0 & 3 & 12.0 & \\
\hline \multicolumn{7}{|l|}{ Residual tumor } \\
\hline No & 37 & 17 & 94.4 & 20 & 80.0 & \multirow[t]{2}{*}{0.178} \\
\hline Yes & 6 & 1 & 5.6 & 5 & 20.0 & \\
\hline \multicolumn{7}{|l|}{ Chemotherapy } \\
\hline None & 6 & 5 & 27.8 & 1 & 4.0 & \multirow[t]{3}{*}{0.0149} \\
\hline Platinum-based & 10 & 6 & 33.3 & 4 & 16.0 & \\
\hline Taxane plus platinum & 27 & 7 & 38.9 & 20 & 80.0 & \\
\hline
\end{tabular}

KIF20A, Kinesin family member 20A; FIGO, International Federation of Gynecology and Obstetrics; CA-125, cancer antigen 125; USO, unilateral salpingooophorectomy; BSO, bilateral salpingooophorectomy. aStandard surgery, including hysterectomy, bilateral salpingo-oophorectomy, with or without omentectomy (OM).
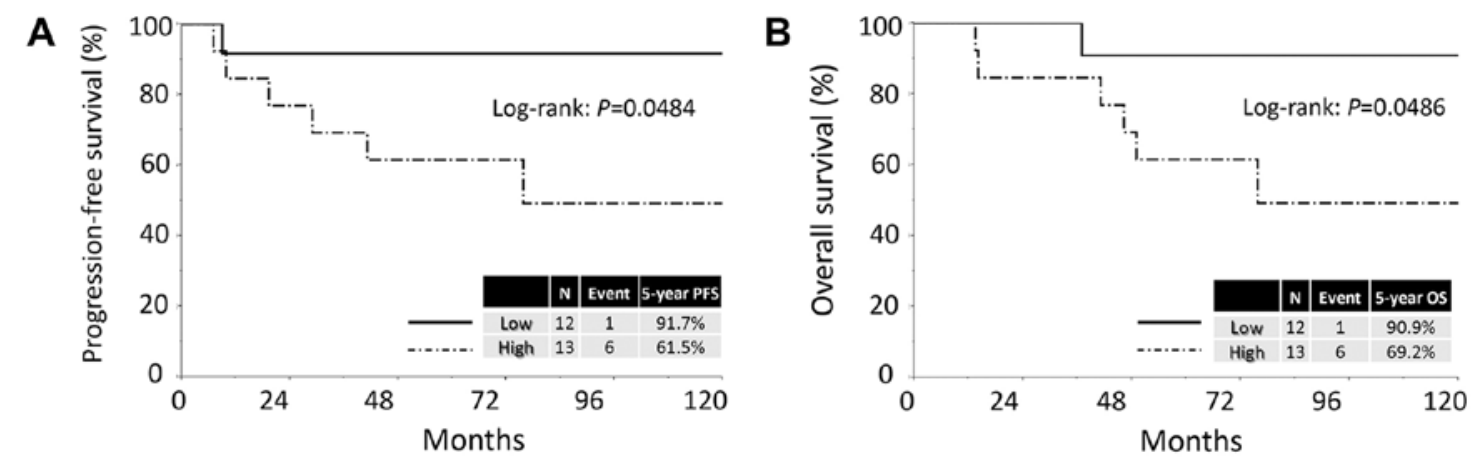

Figure 3. Kaplan-Meier progression-free survival curves for CCC patients with stage I tumors according to the immunoreactivity of KIF20A. (A) PFS. Solid line indicates low KIF20A expression (N=12). Dashed line represents high KIF20A immunoexpression ( $\mathrm{N}=13)$. Patients positive for KIF20A expression showed a significantly poorer PFS ( $\mathrm{P}=0.0484)$. (B) OS. Solid line indicates low KIF20A expression (N=12). Dashed line represents high KIF20A immunoexpression ( $\mathrm{N}=13)$. Patients positive for KIF20A expression showed a significantly poorer PFS ( $\mathrm{P}=0.0486)$. PFS, progression-free survival; OS, overall survival. 


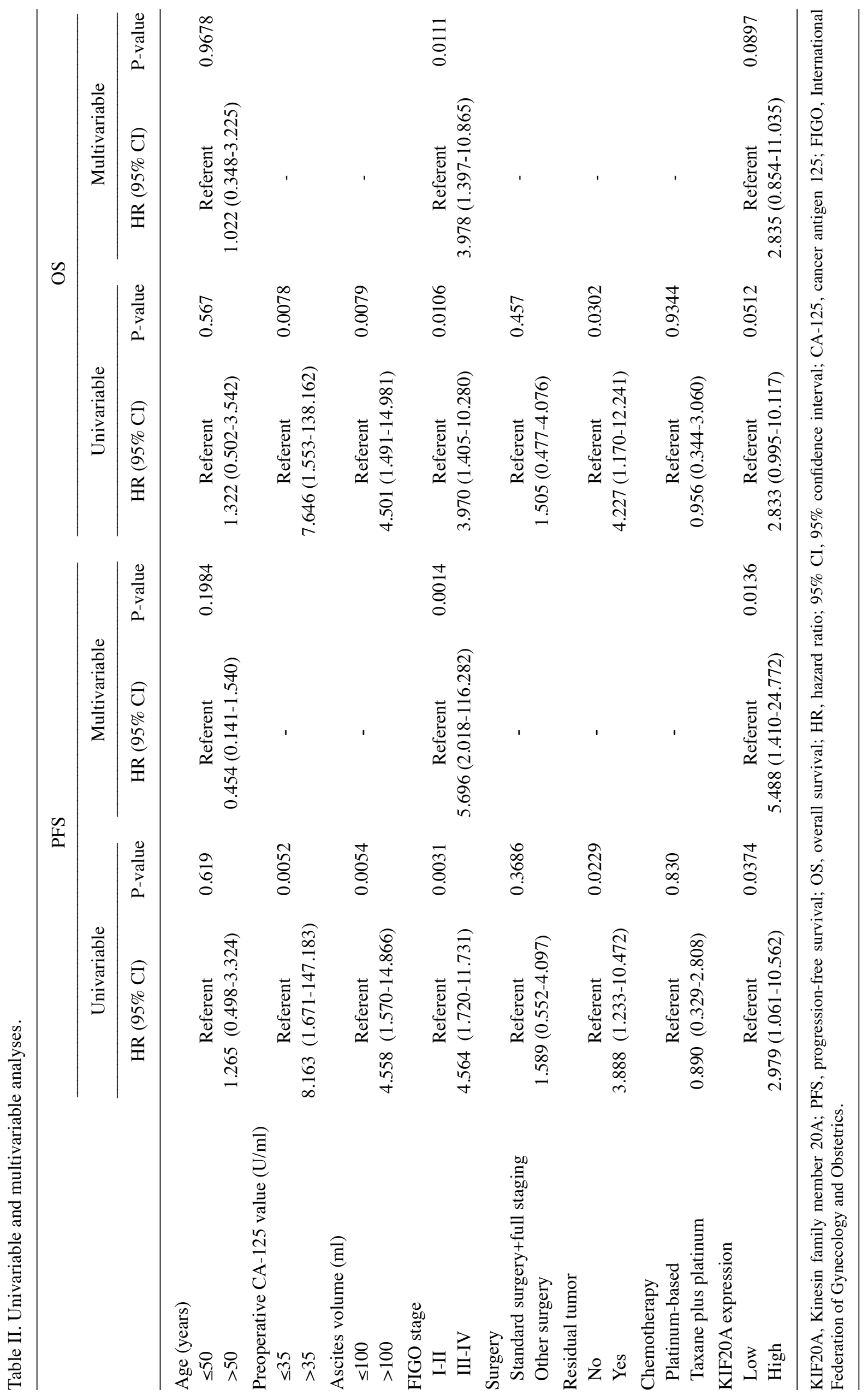




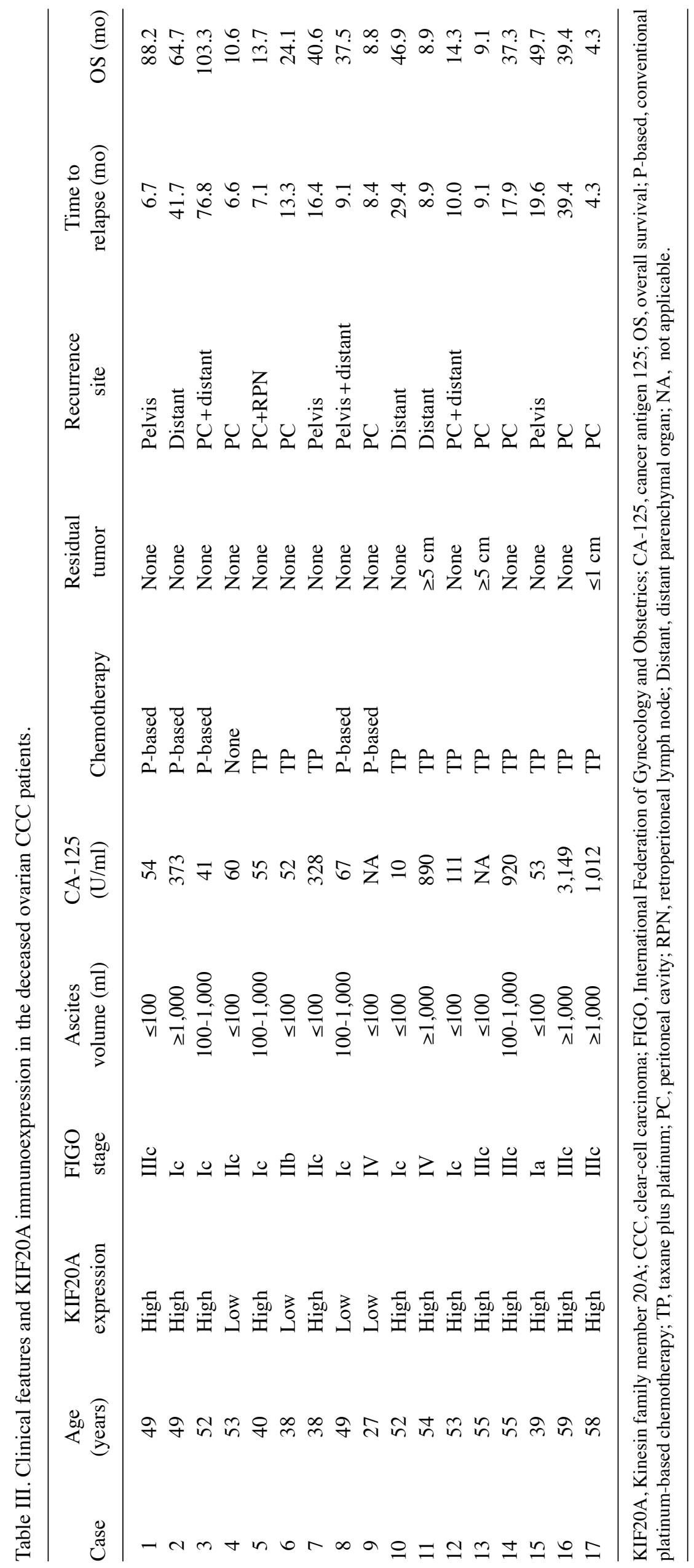


A

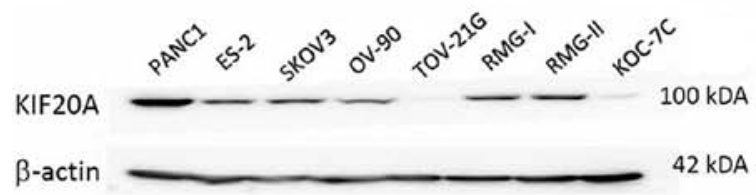

B

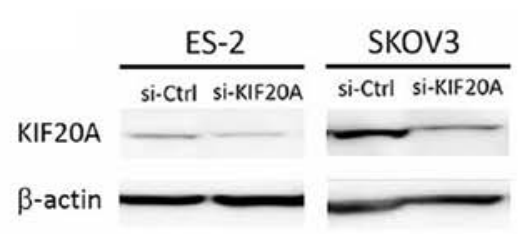

C
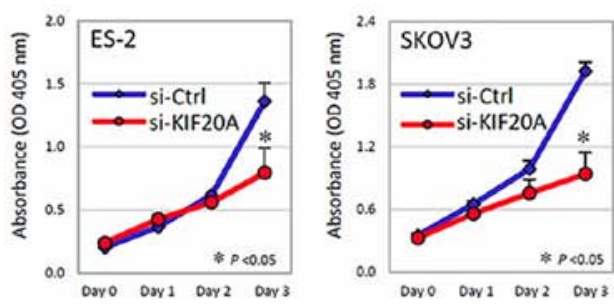

E
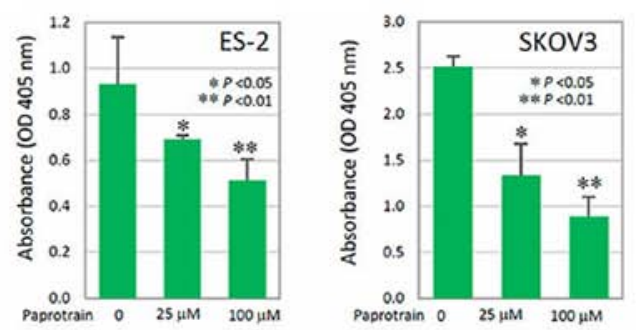
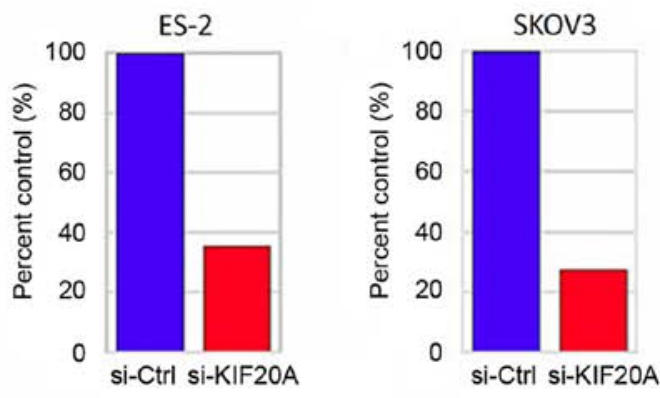

D
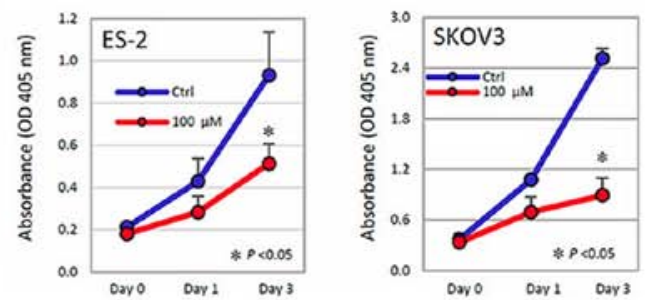

$\mathbf{F}$

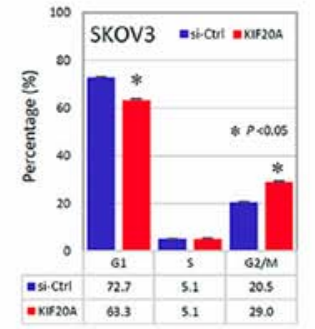

G
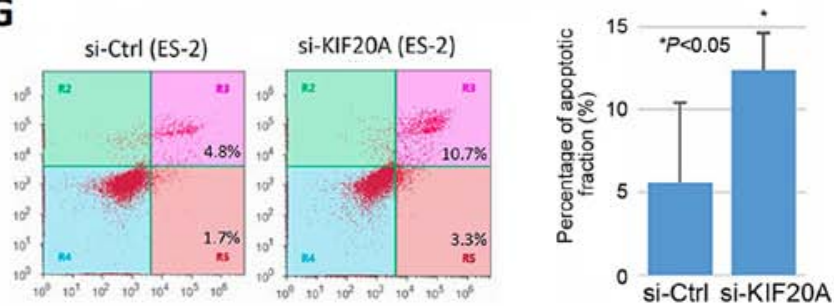

Figure 4. Association between KIF20A expression and the proliferation-promoting effect. (A) The expression of KIF20A in various CCC or non-CCC cell lines. KIF20A was expressed in ES-2, SKOV-3, OV-90, RMG-I and RMG-II cells. In TOV-21G and KOC-7C cells, the expression of KIF20A was weak. PANC1 (pancreatic cancer cells: positive control), ES-2, RMG-I, RMG-II, TOV-21G and KOC-7C cells (CCC cells) and SKOV3, OV-90 (non-CCC ovarian carcinoma cells) were used. (B) Western blotting and densitometric analyses showing the inhibitory efficacy of KIF20A expressions by siRNA (ES-2 and SKOV3). (C) The effect of KIF20A on cell proliferation in ES2 and SKOV3 cells transfected with KIF20A-siRNA (si-KIF20A) or control siRNA (si-ctrl) using Cell Counting Kit-8 (CCK-8). " $\mathrm{P}<0.05$ indicates significance. (D) The proliferation-inhibitory effect of Paprotrain, a cell-permeable acrylonitrile compound that inhibits the kinesin- 6 family member KIF20A (time course). " $\mathrm{P}<0.05$ indicates significance. (E) The proliferation-inhibitory effect of Paprotrain was observed in a concentration-dependent manner. ${ }^{*} \mathrm{P}<0.05,{ }^{* *} \mathrm{P}<0.01$ indicate significance. (F) Evaluation of the cell cycle distribution using flow cytometric analysis. Suppression of KIF20A expression resulted in an increase in the G2/M phase population and a decrease in the G1 phase population, suggesting that knockdown of KIF20A induced G2/M arrest ( $\left.{ }^{*} \mathrm{P}<0.05\right)$. (G) Enhancement of the apoptotic fraction by the downregulation of KIF20A. In the si-KIF20A-transfected cells, the apoptosis-induced fraction was higher than that in the control-siRNA-transfected cells [( $\mathrm{P}<0.05)$, respectively].

in 13 of the 17 patients (76.5\%). In the majority of deceased patients, the most frequent site of recurrence was the peritoneal cavity and/or distant parenchymal organs (14/17: 82.4\%).
Association between KIF20A expression and the proliferation-promoting effect. We subsequently investigated the role of KIF20A in the malignant characteristics of CCC 
cells using several in vitro experiments. We initially examined the expression of KIF20A in various CCC and non-CCC cells. KIF20A was expressed in RMG-I, RMG-II and ES-2 cells, but lower-level expression of KIF20A was observed in KOC-7C and TOV-21G cells (these lines are all CCC cells). As non-CCC/EOC cells, KIF20A was expressed in SKOV-3 and OV-90 cells (Fig. 4A). We further examined whether KIF20A was associated with the proliferation-promoting effect in vitro. ES-2 and SKOV3 cells were successfully transfected with siRNAs (si-KIF20A) (Fig. 4B) and were assessed by the cell proliferation assay (CCK-8 assay) as described above. KIF20A knockdown significantly decreased the cell proliferation (Fig. 4C) $(\mathrm{P}<0.05)$. To further confirm that KIF20A was involved in cell proliferation, we again performed the CCK-8 assay using Paprotrain, which is a cell-permeable acrylonitrile compound that inhibits KIF20A. As a result, treatment with $100 \mu \mathrm{M}$ of Paprotrain led to an $\sim 50 \%$ decrease in the proliferation ability (Fig. 4D) $(\mathrm{P}<0.05)$ in the ES-2 and SKOV3 cells. In addition, we confirmed that this proliferation-inhibitory effect was observed in a concentration-dependent manner (Fig. 4E).

To determine the mitogenic effect of KIF20A, we then performed cell-cycle analysis using ES2 and SKOV3 cells. The distribution of the cell cycle was tested using flow cytometric analysis. As shown in Fig. 4F, the suppression of KIF20A expression led to the increase in the G2/M phase population and decrease in the G1 phase population. This observation suggested that knockdown of KIF20A induced the $\mathrm{G} 2 / \mathrm{M}$ arrest $(\mathrm{P}<0.05)$. We finally tested whether the downregulation of KIF20A expression led to the enhanced apoptotic fraction using the apoptosis assay. Consequently, in the si-KIF20A-transfected ES-2 cells, the apoptosis-induced fraction was higher than that in the the control-siRNA transfected ES-2 cells $[12.4 \pm 2.3$ vs. $5.6 \pm 4.9 \%(\mathrm{P}<0.05)$, respectively] (Fig. 4G). These results indicate that KIF20A may be involved in the mitogenic effect in CCC cells.

\section{Discussion}

In the present study, we first demonstrated that KIF20A expression was significantly correlated with a poor oncologic outcome in patients with ovarian CCC. Furthermore, multivariate analyses demonstrated that a higher expression of KIF20A was an independent prognostic indicator of a poorer PFS of CCC patients. Up to the present, a number of studies have demonstrated the important association between KIF20A expression and aggressive phenotypes in several solid malignancies. Zhang et al (24) demonstrated that elevated KIF20A expression was associated with a number of clinicopathological factors in cervical squamous cell carcinoma, including HPV infection, stage, recurrence, lymphovascular space involvement, nodal status, and poor outcome in patients with this tumor, and concluded that aberrant KIF20A expression is a novel independent unfavorable prognostic indicator that may be a potential therapeutic target for cervical cancer. Liu et al (25) reported that KIF20A was aberrantly expressed in nasopharyngeal cancer, and that high KIF20A protein expression was significantly correlated with poor 5-year OS and PFS. The cumulative 5-year OS and PFS for the high KIF20A-expressing group were 78.5 and $62.7 \%$, respectively, and 95.9 and $90.8 \%$, respectively, for the low or no
KIF20A-expressing group. In addition, Duan et al (31) reported the clinical effect of KIF20A expression in 119 patients with glioma. They demonstrated that patients with positive KIF20A expression showed significantly poorer OS compared with patients with negative KIF20A expression (median, 16.0 vs. 39.0 months, respectively). Our current results are consistent with those of these previous studies. Similarly to patients with EOC, those with CCC show an unfavorable prognosis, principally attributable to its asymptomatic intraperitoneal dissemination with or without distant metastases to parenchymal organs (32). Our data were obtained from a small number of patients, and therefore, the prognostic significance of KIF20 needs to be confirmed in a larger number of patients. At least, the present findings clarify that the immunoreactive expression of KIF20A may be a critical indicator of a poor prognosis in CCC patients, and its identification may result in the selection of better strategic options.

Our current data indicated that KIF20A participated in the growth of CCC cells. Furthermore, we demonstrated that KIF20A regulates cell division, and the knockdown of KIF20A induced apoptosis and cell cycle arrest via nuclear localization. In addition, the present study showed that the inhibition of KIF20A induced the multinucleation of cells, which ensures the inhibition of cell division. KIF20A is required during mitosis exit for the final step of cytokinesis, and its inhibitor, Paprotrain, inhibits the recruitment of the surviving chromosome passenger proteins and aurora B to the central spindle during the anaphase (33). A previous report demonstrated that KIF20A plays a crucial role in the proliferation and tumor growth of hepatocellular carcinoma as a novel downstream target of glioma-associated oncogene 2 (Gli2) (26). This evidence prompted us to hypothesize that the growth-inhibitory effect in KIF20A silencing CCC cells may be partly attributable to the inhibition of activity to drive the cell cycle. Moreover, tumor invasion and migration are representative steps in the peritoneal dissemination of this tumor $(34,35)$. Stangel et al (15) reported that KIF20A silencing with small interfering RNA molecules resulted in the inhibition of motility and invasion of pancreatic cancer cell lines, as well as the growth-promoting effect of KIF20A. It is possible that KIF20A is involved in these multiple steps of the peritoneal metastasis of CCC. We hope to clarify these unelucidated mechanisms of KIF20A in CCC in our next investigation.

Additionally, CCC patients generally showed a very low chemoresponse rate to platinum-based compounds, leading to intrinsic chemoresistance (9-36). In the present study, the majority of patients showing a higher expression of KIF20A died of the disease [13/17 (76.4\%)]. In general, the remaining chemoresistant clone may be a cause of the high rate of recurrence and/or cancer-related mortality. Thus, one of the mainstream mechanisms leading to patients with KIF20A expression showing an unfavorable clinical outcome may be based on the chemoresistant hallmark as well as the growth-promoting effect of KIF20A. Actually, Khongkow et al (22) revealed that paclitaxel targets the FOXM1-KIF20A axis to drive abnormal mitotic spindle formation and mitotic catastrophe, and that deregulations of FOXM1 and KIF20A expression may be involved in the resistance to paclitaxel. Based on our current result that silencing KIF20A expression induced the 
upregulation of apoptosis in CCC cells, its knockdown may lead to enhancement of the chemosusceptibility of this tumor, although we did not investigate the direct correlation between KIF20A expression and chemoresistance. Also, we hope to verify this hypothesis in the next study in order to clarify the CCC-specific biological hallmarks.

To the best of our knowledge, the present study is the first to report that the expression of KIF20A was closely associated with a poor oncologic outcome of patients with CCC. The current findings may be based on the growthand/or chemoresistant-promoting effects of KIF20A, although further investigation is needed to clarify its molecular mechanism. Furthermore, KIF20A could be considered as an ideal cancer-testis antigen and the KIF20A peptide may be a cancer vaccine for pancreatic cancer. A phase I/II clinical trial using a peptide vaccine derived from KIF20A revealed that patients with advanced pancreatic cancer vaccinated with KIF20A-derived peptide had a better prognosis than control patients $(37,38)$. From this point of view, the present study demonstrated the possibility of using KIF20A-derived peptide vaccine for the treatment of advanced CCC.

In summary, we identified KIF20A as a prognostic indicator and therapeutic target in patients with CCC. However, the detailed functions of KIF20A remain unclear. Particularly, we think that the present study was preliminary because overexpression or restoration assays and animal experiments have not been performed to conclude the possible implication of KIF20A in the proliferation of CCC. Further studies are needed in order to realize therapeutic application. We believe that the mechanistic clarification of KIF20A will help improve treatment for $\mathrm{CCC}$ patients by adding criteria for the administration of systematic therapy in the future.

\section{Acknowledgements}

The authors thank Mrs. Mai Sugiyama for her technical support.

\section{Funding}

The present study was supported by the Japan Society for the Promotion of Science (JSPS) Grant-in-Aid for Scientific Research (24592512 and 15K10714 to KS).

\section{Availability of data and materials}

The datasets used during the present study are available from the corresponding author upon reasonable request.

\section{Authors' contributions}

KS and TS conceived and designed the study. YK performed the experiments. YK and HK wrote the manuscript. KS reviewed and edited the manuscript. SS, FU, KN and RS performed data acquisition and curation. Supervision throughout this manuscript was done by FK and TS. All authors read and approved the manuscript and agree to be accountable for all aspects of the research in ensuring that the accuracy or integrity of any part of the work are appropriately investigated and resolved.

\section{Ethics approval and consent to participate}

Patient's consent protocol was approved by the Ethics Committee of Nagoya University (Nagoya, Japan).

\section{Consent for publication}

Not applicable.

\section{Competing interests}

The authors declare that they have no competing interests.

\section{References}

1. Kikkawa F, Nawa A, Ino K, Shibata K, Kajiyama $H$ and Nomura S: Advances in treatment of epithelial ovarian cancer. Nagoya J Med Sci 68: 19-26, 2006.

2. Brun JL, Feyler A, Chêne G, Saurel J, Brun G and Hocké C: Long-term results and prognostic factors in patients with epithelial ovarian cancer. Gynecol Oncol 78: 21-27, 2000.

3. Chan JK, Teoh D, Hu JM, Shin JY, Osann K and Kapp DS: Do clear cell ovarian carcinomas have poorer prognosis compared to other epithelial cell types? A study of 1411 clear cell ovarian cancers. Gynecol Oncol 109: 370-376, 2008.

4. Ryu SY, Park SI, Nam BH, Kim I, Yoo CW, Nam JH, Lee KH, Cho $\mathrm{CH}$, Kim JH, Park SY, et al: Prognostic significance of histological grade in clear-cell carcinoma of the ovary: A retrospective study of Korean gynecologic oncology group. Ann Oncol 20: 1032-1036, 2009.

5. Gynecology JSoOa: Annual patient report. Acta Obstet Gynaecologia Japonica 68: 1117-1160, 2016.

6. Jenison EL, Montag AG, Griffiths CT, Welch WR, Lavin PT, Greer J and Knapp RC: Clear cell adenocarcinoma of the ovary: A clinical analysis and comparison with serous carcinoma. Gynecol Oncol 32: 65-71, 1989.

7. Kennedy AW, Biscotti CV, Hart WR and Webster KD: Ovarian clear cell adenocarcinoma. Gynecol Oncol 32: 342-349, 1989.

8. Satoh T, Oki A, Uno K, Sakurai M, Ochi H, Okada S, Minami R, Matsumoto K, Tanaka YO, Tsunoda $\mathrm{H}$, et al: High incidence of silent venous thromboembolism before treatment in ovarian cancer. Br J Cancer 97: 1053-1057, 2007.

9. Sugiyama T, Kamura T, Kigawa J, Terakawa N, Kikuchi Y, Kita T, Suzuki M, Sato I and Taguchi K: Clinical characteristics of clear cell carcinoma of the ovary: A distinct histologic type with poor prognosis and resistance to platinum-based chemotherapy. Cancer 88: 2584-2589, 2000.

10. Mizuno M, Kikkawa F, Shibata K, Kajiyama H, Ino K, Kawai M, Nagasaka T and Nomura S: Long-term follow-up and prognostic factor analysis in clear cell adenocarcinoma of the ovary. J Surg Oncol 94: 138-143, 2006.

11. Yan GR, Zou FY, Dang BL, Zhang Y, Yu G, Liu X and He QY: Genistein-induced mitotic arrest of gastric cancer cells by downregulating KIF20A, a proteomics study. Proteomics 12: 2391-2399, 2012.

12. Zhang Y, Liu J, Peng X, Zhu CC, Han J, Luo J and Rui R: KIF20A regulates porcine oocyte maturation and early embryo development. PLoS One 9: e102898, 2014.

13. Echard A, Jollivet F, Martinez O, Lacapere JJ, Rousselet A, Janoueix-Lerosey I and Goud B: Interaction of a Golgi-associated kinesin-like protein with Rab6. Science 279: 580-585, 1998.

14. Lai F, Fernald AA, Zhao N and Le Beau MM: cDNA cloning, expression pattern, genomic structure and chromosomal location of RAB6KIFL, a human kinesin-like gene. Gene 248: 117-125, 2000.

15. Stangel D, Erkan M, Buchholz M, Gress T, Michalski C, Raulefs S, Friess $\mathrm{H}$ and Kleeff $\mathrm{J}$ : Kif20a inhibition reduces migration and invasion of pancreatic cancer cells. J Surg Res 197: 91-100, 2015.

16. Ho JR, Chapeaublanc E, Kirkwood L, Nicolle R, Benhamou S, Lebret T, Allory Y, Southgate J, Radvanyi F and Goud B: Deregulation of Rab and Rab effector genes in bladder cancer. PLoS One 7: e39469, 2012

17. Imai K, Hirata S, Irie A, Senju S, Ikuta Y, Yokomine K, Harao M, Inoue M, Tomita Y, Tsunoda T, et al: Identification of HLA-A2-restricted CTL epitopes of a novel tumour-associated antigen, KIF20A, overexpressed in pancreatic cancer. Br J Cancer 104: 300-307, 2011. 
18. Kikuchi T, Daigo Y, Katagiri T, Tsunoda T, Okada K, Kakiuchi S, Zembutsu H, Furukawa Y, Kawamura M, Kobayashi K, et al: Expression profiles of non-small cell lung cancers on cDNA microarrays: Identification of genes for prediction of lymph-node metastasis and sensitivity to anti-cancer drugs. Oncogene 22: 2192-2205, 2003.

19. Taniuchi K, Furihata M and Saibara T: KIF20A-mediated RNA granule transport system promotes the invasiveness of pancreatic cancer cells. Neoplasia 16: 1082-1093, 2014.

20. Gasnereau I, Boissan M, Margall-Ducos G, Couchy G, Wendum D, Bourgain-Guglielmetti F, Desdouets C, Lacombe ML, Zucman-Rossi J and Sobczak-Thépot J: KIF20A mRNA and its product MKlp2 are increased during hepatocyte proliferation and hepatocarcinogenesis. Am J Pathol 180: 131-140, 2012.

21. Exertier P, Javerzat S, Wang B, Franco M, Herbert J, Platonova N, Winandy M, Pujol N, Nivelles O, Ormenese S, et al: Impaired angiogenesis and tumor development by inhibition of the mitotic kinesin Eg5. Oncotarget 4: 2302-2316, 2013.

22. Khongkow P, Gomes AR, Gong C, Man EP, Tsang JW, Zhao F, Monteiro LJ, Coombes RC, Medema RH, Khoo US and Lam EW: Paclitaxel targets FOXM1 to regulate KIF20A in mitotic catastrophe and breast cancer paclitaxel resistance. Oncogene 35: 990-1002, 2016

23. Zou JX, Duan Z, Wang J, Sokolov A, Xu J, Chen CZ, Li JJ and Chen HW: Kinesin family deregulation coordinated by bromodomain protein ANCCA and histone methyltransferase MLL for breast cancer cell growth, survival, and tamoxifen resistance. Mol Cancer Res 12: 539-549, 2014.

24. Zhang W, He W, Shi Y, Gu H, Li M, Liu Z, Feng Y, Zheng N, Xie C and Zhang Y: High expression of KIF20A is associated with poor overall survival and tumor progression in early-stage cervical squamous cell carcinoma. PLoS One 11: e0167449, 2016.

25. Liu SL, Lin HX, Qiu F, Zhang WJ, Niu CH, Wen W, Sun XQ, Ye LP, Wu XQ, Lin CY, et al: Overexpression of Kinesin family member $20 \mathrm{~A}$ correlates with disease progression and poor prognosis in human nasopharyngeal cancer: A retrospective analysis of 105 patients. PLoS One 12: e0169280, 2017.

26. Shi C, Huang D, Lu N, Chen D, Zhang M, Yan Y, Deng L, Lu Q, $\mathrm{Lu} \mathrm{H}$ and Luo S: Aberrantly activated Gli2-KIF20A axis is crucial for growth of hepatocellular carcinoma and predicts poor prognosis. Oncotarget 7: 26206-26219, 2016.

27. Hosono S, Kajiyama H, Terauchi M, Shibata K, Ino K, Nawa A and Kikkawa F: Expression of Twist increases the risk for recurrence and for poor survival in epithelial ovarian carcinoma patients. Br J Cancer 96: 314-320, 2007.

28. Zeppernick F and Meinhold-Heerlein I: The new FIGO staging system for ovarian, fallopian tube, and primary peritoneal cancer. Arch Gynecol Obstet 290: 839-842, 2014.
29. Chen VW, Ruiz B, Killeen JL, Cote TR, Wu XC and Correa CN: Pathology and classification of ovarian tumors. Cancer 97: 2631-2642, 2003.

30. Sakata J, Kajiyama H, Suzuki S, Utsumi F, Niimi K, Sekiya R, Shibata K, Senga T and Kikkawa F: Impact of positive ZEB1 expression in patients with epithelial ovarian carcinoma as an oncologic outcome-predicting indicator. Oncol Lett 14: 4287-4293, 2017.

31. Duan J, Huang W and Shi H: Positive expression of KIF20A indicates poor prognosis of glioma patients. Onco Targets Ther 9: 6741-6749, 2016.

32. Kajiyama H, Shibata K, Mizuno M, Yamamoto E, Fujiwara S, Umezu T, Suzuki S, Nakanishi T, Nagasaka T and Kikkawa F: Postrecurrent oncologic outcome of patients with ovarian clear cell carcinoma. Int J Gynecol Cancer 22: 801-806, 2012.

33. Tcherniuk S, Skoufias DA, Labriere C, Rath O, Gueritte F, Guillou C and Kozielski F: Relocation of Aurora B and survivin from centromeres to the central spindle impaired by a kinesin-specific MKLP-2 inhibitor. Angew Chem Int Ed Engl 49: 8228-8231, 2010

34. Kajiyama H, Shibata K, Terauchi M, Ino K, Nawa A and Kikkawa F: Involvement of SDF-1alpha/CXCR4 axis in the enhanced peritoneal metastasis of epithelial ovarian carcinoma. Int J Cancer 122: 91-99, 2008.

35. Terauchi M, Kajiyama H, Yamashita M, Kato M, Tsukamoto $H$, Umezu T, Hosono S, Yamamoto E, Shibata K, Ino K, et al: Possible involvement of TWIST in enhanced peritoneal metastasis of epithelial ovarian carcinoma. Clin Exp Metastasis 24: 329-339, 2007.

36. Shimada M, Kigawa J, Ohishi Y, Yasuda M, Suzuki M, Hiura M, Nishimura R, Tabata T, Sugiyama T and Kaku T: Clinicopathological characteristics of mucinous adenocarcinoma of the ovary. Gynecol Oncol 113: 331-334, 2009.

37. Suzuki N, Hazama S, Ueno T, Matsui H, Shindo Y, Iida M, Yoshimura K, Yoshino S, Takeda K and Oka M: A phase I clinical trial of vaccination with KIF20A-derived peptide in combination with gemcitabine for patients with advanced pancreatic cancer. J Immunother 37: 36-42, 2014

38. Asahara S, Takeda K, Yamao K, Maguchi H and Yamaue H: Phase I/II clinical trial using HLA-A24-restricted peptide vaccine derived from KIF20A for patients with advanced pancreatic cancer. J Transl Med 11: 291, 2013.

This work is licensed under a Creative Common Attribution-NonCommercial-NoDerivatives 4.0 International (CC BY-NC-ND 4.0) License. 